Helbig, Marko; Dahlke, Katja; Hilger, Ingrid; Kmec, Martin; Sachs, Jürgen:

\title{
UWB microwave imaging of heterogeneous breast phantoms
}

Zuerst erschienen in: Biomedical Engineering = Biomedizinische Technik. - Berlin [u.a.] : de Gruyter. - 57 (2012), Suppl. 1, Track-B, p. 486-489.

Erstveröffentlichung: $\quad$ 2012-08-24

ISSN (online): $\quad$ 1862-278X

ISSN (print): $\quad$ 0013-5585

DOI: $\quad 10.1515 /$ bmt-2012-4237

[Zuletzt gesehen: 2019-08-12]

„Im Rahmen der hochschulweiten Open-Access-Strategie für die Zweitveröffentlichung identifiziert durch die Universitätsbibliothek IImenau."

"Within the academic Open Access Strategy identified for deposition by Ilmenau University Library."

„Dieser Beitrag ist mit Zustimmung des Rechteinhabers aufgrund einer (DFGgeförderten) Allianz- bzw. Nationallizenz frei zugänglich."

"This publication is with permission of the rights owner freely accessible due to an Alliance licence and a national licence (funded by the DFG, German

Research Foundation) respectively." 


\title{
UWB microwave imaging of heterogeneous breast phantoms
}

\author{
M. Helbig ${ }^{1}$, K. Dahlke ${ }^{2}$, I. Hilger ${ }^{2}$, M. Kmec ${ }^{1}$, J. Sachs ${ }^{1}$ \\ ${ }^{1}$ Institute for Information Technology, Ilmenau University of Technology, 98684 Ilmenau, Germany \\ ${ }^{2}$ Institute of Diagnostic and Interventional Radiology, Jena University Hospital - FSU Jena, 07747 Jena, Germany
}

\begin{abstract}
UWB microwave imaging has potential for early-stage screening diagnostics of breast cancer. We present an experimental measurement setup for breast phantom trials based on M-sequence radar technology and active small dipole antennas as pre-stage for real in vivo measurements in a prone examination position. We describe current development steps as well as first experiences concerning the imaging of heterogeneous breast phantoms.
\end{abstract}

\section{Introduction}

The application of microwave ultra-wideband (UWB) technologies for biomedical imaging is motivated by the contrast of electrical properties between healthy and cancerous tissue. Caused by the non-ionizing nature of electromagnetic waves in the microwave frequency range and the potential for a cost-efficient imaging technology it represents a promising alternative for early-stage screening diagnostics of breast cancer in addition to mammography or MRI.

Numerous research groups are working in this field since more than one decade [1]-[3]. The challenges which have to be overcome concerning real measurements are multifaceted and depend on the conditions of the measurement scenario.

We present an experimental measuring setup using breast models in order to approximate in vivo measurement scenarios, with the patient in a prone examination position. The antennas are arranged in quasi direct contact with the breast behind an approximately hemispherical examination mold, to which the breast will adhere during the measurement.

Continuing our last publications [4]-[7], this paper describes technical improvements of our measurement approach and first experience with imaging of heterogeneous phantoms.

\section{$2 \quad$ Measurement setup}

\subsection{Radar device}

For our measurements we use M-sequence radar technology developed at Ilmenau University of Technology. The pseudo-noise stimulation signals (M-sequences) can be generated quite simply by high-speed digital shift registers. This promotes monolithic system integration by low cost semi-conductor technologies and can be used to build very flexible and time stable (low jitter and drift) UWB-sensor systems as described e.g. in [8].

This measurement approach deals with continuous wave signals which distribute the signal energy equally over time, thus, the signal magnitudes remain low. The reduced voltage exposure of the medium under test makes this technology suitable for medical applications (spectroscopy and imaging), e.g. for breast cancer detection. For the measurements we use a base-band system (bandwidth $9 \mathrm{GHz}$ ) containing 2 transmitters (Tx) and 4 receivers (Rx).

\subsection{Antennas and antenna array}

The efficient penetration of the electromagnetic waves into the material under test and the high spatial resolution of the reflected signals are crucial issues for the measurement setup. An efficient antenna array design concerning biomedical UWB imaging purposes have to care for shape and duration of signal impulses, angle dependency of the impulse response, and physical dimensions of the antenna.

We pursue the approach of short active broadband dipoles. The antennas are implemented on Rogers ${ }^{\circledR} 4003$ substrate $(0.5 \mathrm{~mm})$ using PCB technology. The dimensions of the bow-ties are $8 \mathrm{~mm} \times 4 \mathrm{~mm}$. They are differentially fed, and the balanced feeding is realized by differential amplifier circuits [9].

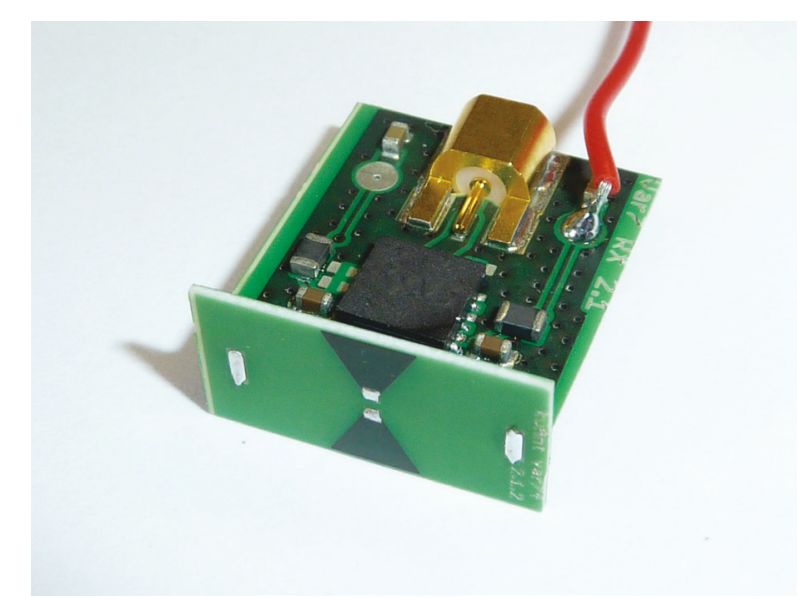

Figure 1. Active antenna element $(\mathrm{Rx})$ with the $8 \mathrm{~mm}$ bow-tie dipole and the amplifier circuit board. 
These antennas cannot be matched over a large bandwidth. In order to avoid unwanted reflections between antenna and amplifier, the amplifier is placed directly at the antenna feed point (Fig. 1). In this way the feeding of the active antenna element can be done single ended which halves the number of feeding cables.

A slide-in mounting system was designed which allows flexible antenna application and replacement and, therefore, facilitates investigations of various $\mathrm{Rx}-\mathrm{Tx}-$ arrangements. The mounting system used at present has a holding capacity for 28 antennas (Fig. 2).

The contact between antennas and breast skin represents a crucial aspect for sufficient signal quality. Regarding clinical requirements (e.g. disinfection) we plan to place the antennas behind a thin cleanable examination mold. But this additional interface reduces the signal quality significantly. Therefore, a thin $(\sim 2 \mathrm{~mm})$ matching layer consisting of material with higher permittivity than the mold material (plastic or glass) is inserted between mold and antennas, in order to increase the signal energy penetrating into the tissue and to reduce the backward radiation. Other requirements for this layer material are their physical properties (flexibility) and long term chemical stability. We propose polymer-powder composites, where dielectric powders (e.g. carbon meal or barium titanate powder) will be admixed to silicone rubber (Fig. 2).

\section{$3 \quad$ Breast phantoms}

The phantoms are tissue mimicking oil-gelatin phantoms according to [10]. The dielectric properties can be adjusted by means of the oil content. For our measurements we use several types of material: $40 \%$ oil content material mimics healthy tissue, which approximately corresponds to group II of adipose-defined tissue (31\%-84\% adipose tissue) according to [11]. The $0 \%$ and $10 \%$ oil content material simulates tumor tissue. Other concentrations $(20 \%, 30 \%, 50 \%$ and $60 \%$ oil content) are used to imitate heterogeneous structures like fat or glandular tissue. Further details on the frequency dependent dielectric properties of each material can be found in [10].

In order to realize an optimal contact to the antenna array the phantom material is filled in plastic containers (Fig. 2) identical to the one used as examination mold (diameter $9.5 \mathrm{~cm}, \sim 340 \mathrm{ml}$ ). The containers are hermetically sealed and stored in a refrigerator to avoid chemical instability of the phantom material. The phantoms have to be acclimatized at least 3 hours before starting the measurements.

\section{$4 \quad$ Imaging}

The results shown in this section concern $2 \mathrm{D}$ imaging based on the introduced measurement setup using 8 horizontally aligned antennas (4 transmitters and 4 receivers) at the tumor plane. Because the resulting 16 bi-static signal channels are insufficient for high resolution imaging, we have to consider mechanical scanning as interim solution to achieve sufficient information. In order to simulate antenna rotation, the phantoms are rotated in steps of $11.25^{\circ}$, achieving the potential for 512 signals (16 channels $\mathrm{x} 32$ rotations) to include into the imaging process. However, channels with too wide angle between the boresights of Rx and Tx should be excluded in order to avoid excessive point spreading in the image [5]. Therefore and depending on the geometric arrangement of the antennas within the array, the imaging will practically be comprised of less than all received signals.

We carry out the image processing in the time domain. First we remove clutter which is mainly caused from antenna crosstalk. For every individual antenna constellation, it can be considered as similar during all 32 rotations while the tumor response will vary. Hence, it can be excluded by subtracting the average of all 32 measurements per channel. After that, time domain beamforming can be executed. It can be stated by the following beamformer formula

$$
I\left(\mathbf{r}_{0}\right)=\left(\sum_{n=1}^{N} \sum_{\tau_{W}=-T_{w} / 2}^{T_{w} / 2} w_{n}\left(\tau_{w}, \mathbf{r}_{0}\right) \cdot S_{n}\left(t+\tau_{n}\left(\mathbf{r}_{0}\right)+\tau_{w}\right)\right)^{2}
$$

where $\mathrm{N}$ is the number of channels, $S_{n}(t)$ is the clutter subtracted measurement signal, $\mathbf{r}_{0}$ symbolizes the coordinates of the focal point, $\tau_{n}\left(\mathbf{r}_{0}\right)$ is the focal point dependent time delay of channel $n, w_{n}\left(\tau_{w}, \mathbf{r}_{0}\right)$ is a FIR filter to equalize path dependent dispersion and attenuation (which can be in the simplest case only a weight coefficient) and $I\left(\mathbf{r}_{0}\right)$ is the back scattered energy which has to be mapped over all focal points.
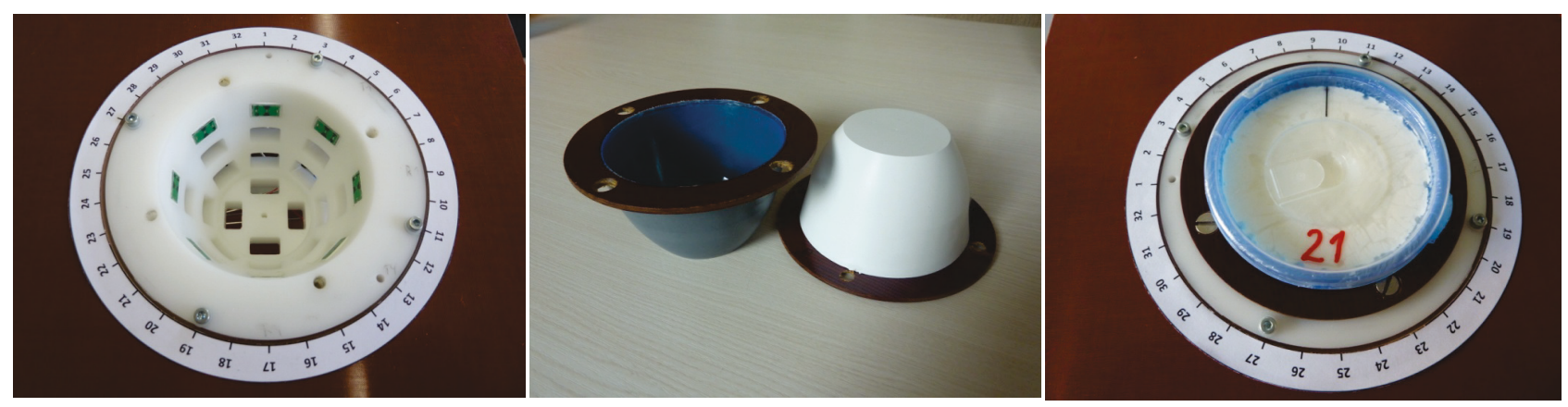

Figure 2. Antenna array (slide-in system) for 28 antennas equipped with the upper 8 antennas (left), two samples of cast contact layers (middle) and the finished measurement setup including a rotatable breast phantom 

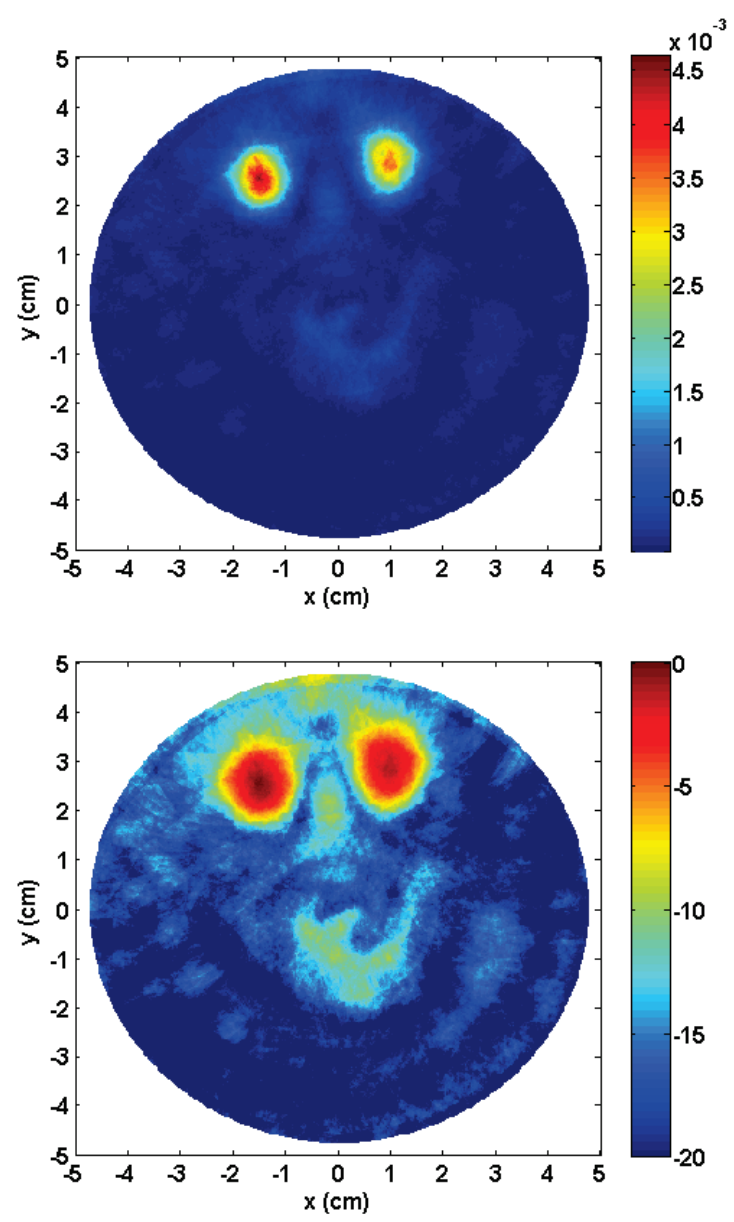

Figure 3. UWB image illustrating the detection and discrimination between two $15 \mathrm{~mm}$ tumor surrogates $(10 \%$ oil content) separated by $30 \mathrm{~mm}$. The images represent the reflected signal strength with linear scale (top) and logarithmic scale in $\mathrm{dB}$ (bottom).
The detection capability of single tumor surrogates within homogeneous healthy tissue mimicking phantom material (40\% oil content) was already shown in [4] and [5]. Fig. 3 shows the resolution performance in order to distinguish between multiple tumors. It indicates that 2 tumors (15 $\mathrm{mm}$ spheres, $10 \%$ oil content, $30 \mathrm{~mm}$ distance between both) can clearly be identified and separated. This result is based on the appropriate time domain signal characteristic (short impulse response with low ringing) provided by the applied short dipole antennas [6].

The following figures represent images of heterogeneous, and thus, more realistic breast phantoms. Fig. 4 shows the reconstruction result of a rosette shaped tumor simulation ( $10 \%$ oil content) and reveals the challenges concerning the exact reconstruction of the whole tumor shape. The outer side is relatively well recognizable, whereas the inner parts are largely blurred. This is due to the high path losses caused from the long propagation path illuminating from the right and due to the tumor caused reflection losses for the waves coming from the left.

Furthermore, we investigated the influence of additional scattering particles mimicking the heterogeneity of real breast tissue. Additionally to the tumor surrogate $(15 \mathrm{~mm}$ gelatin sphere, $0 \%$ oil content) and the surrounding material $(40 \%$ oil content), a third component (3-5 mm crumbs) of different permittivity $(20 \%, 30 \%, 50 \%$, or $60 \%$ oil content, respectively) was inserted into the phantoms. The findings of these first measurements are twofold. On the one hand, concerning the tumor detection the imaging works robustly as the tumors are clearly detectable in all four cases. Two examples are shown in Fig. 5. On the other hand, the low influence of the additional particles reveals the low resolution and, therefore, the need of improvement, because in the presented images the inserted particles are currently not detectable.
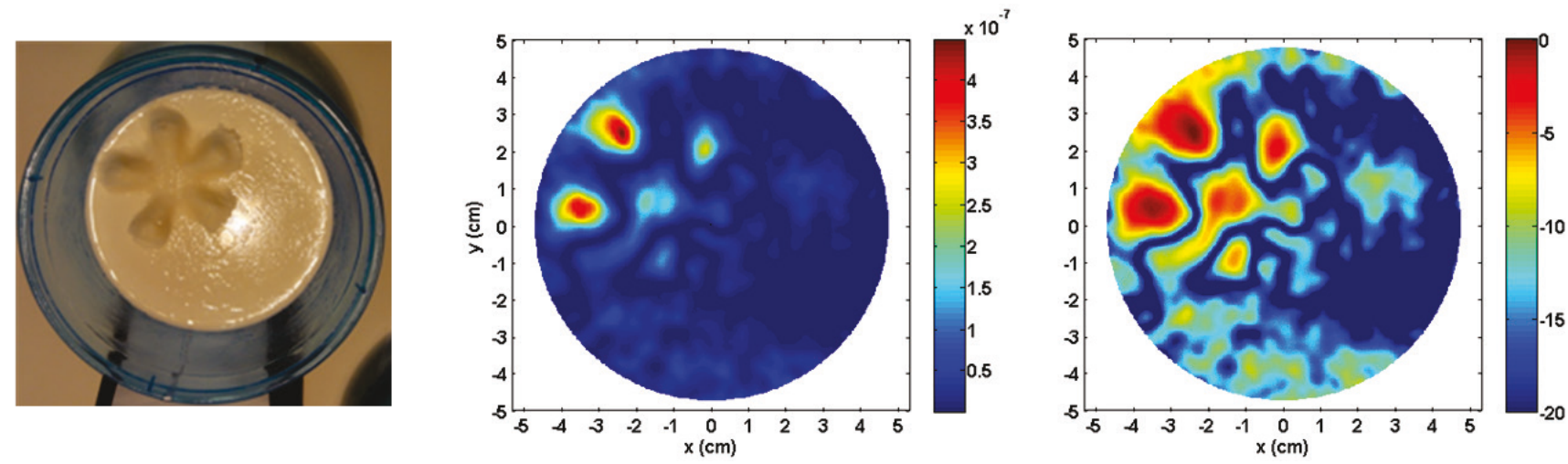

Figure 4. UWB image of a rosette shaped tumor surrogate: Photograph of the phantom before casting the resist layer (left), and images of the reflected signal energy with linear scale (middle) and logarithmic scale in $\mathrm{dB}$ (right) 

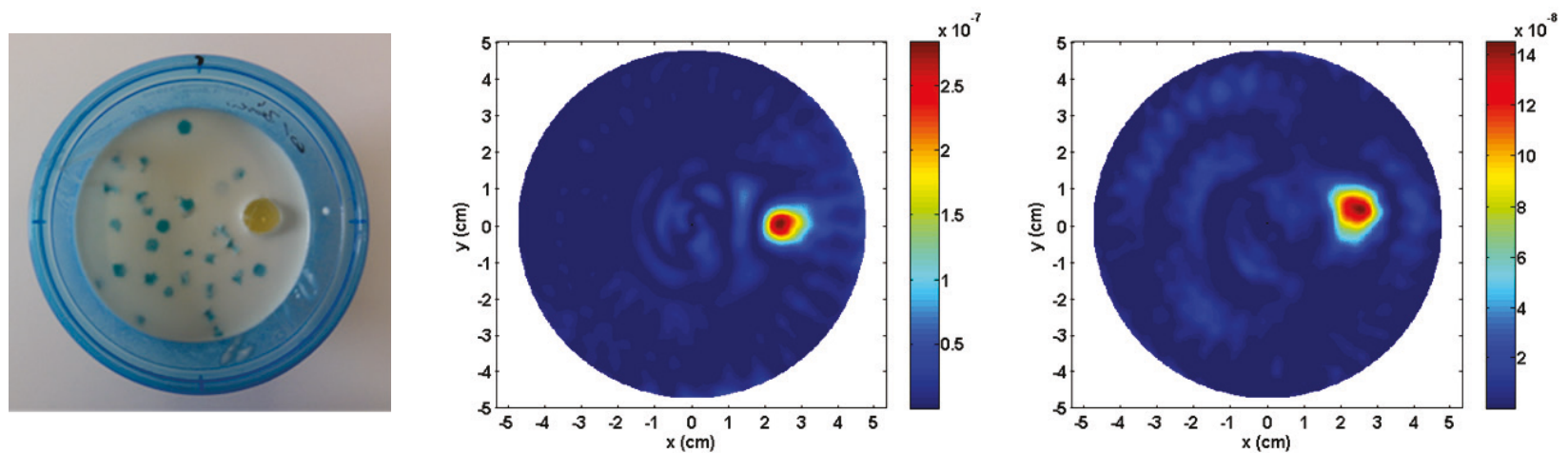

Figure 5. Imaging of heterogeneous breast phantoms: Photograph of a phantom before casting the resist layer (left), UWB images of two samples including scattering crumbs of $20 \%$ oil content (middle) and $50 \%$ oil content (right)

\section{Conclusions}

We introduced an experimental measurement setup for UWB breast imaging using small active antennas (interfacial dipoles) in quasi direct contact to the breast. The capability of this approach was verified by phantom measurements. In comparison to our last publications we gained first experiences with heterogeneous phantoms. The results confirm that the setup is also capable to image more complex measurement scenarios than only single spherical tumor surrogates within a homogeneous environment.

However, the artificial phantom rotation represents a simplification which is not consistent with the system envisaged for in vivo measurements and has to be avoided by means of a MIMO system providing a sufficient number of channels. A radar system (bandwidth $6.5 \mathrm{GHz}$ ) containing 8 transmitters and 16 receivers is built up and currently under investigation for this task. Because this radar system supports inherently 128 signal channels without antenna or phantom rotation, the measurement time will be reduced significantly.

The development of robust calibration procedures for this MIMO system inclusive the estimation of the unknown mean propagation velocity of a real breast under investigation and further resolution enhancement represent the remaining challenges for our future work.

\section{References}

[1] Klemm IJ, Craddock JA, Leendertz A, et al.: "Radarbased breast cancer detection using a hemispherical antenna array - experimental results," IEEE Transactions on Antennas and Propagation, vol. 57, 2009, 1692-1704

[2] Fear EC, Sill J, Stuchly MA: "Experimental feasibility study of confocal microwave imaging for breast tumor detection," IEEE Transactions on Microwave Theory and Techniques, vol. 51, 2003, 887-892

[3] Li X, Davis KS, Hagness SC, et al.: "Microwave imaging via space-time beamforming: Experimental investigation of tumor detection in multi-layer breast phantoms," IEEE Transactions on Microwave Theory and Techniques, vol. 52, no. 8, 2004, 1856-1865.
[4] Helbig M, Hilger I, Kmec M, et al.: "Experimental phantom trials for UWB breast cancer detection," Proc. German Microwave Conference (GeMiC), Ilmenau, 12-14 March 2012.

[5] Helbig M, Kmec M, Sachs J, et al.: "Aspects of Antenna Array Configuration for UWB Breast Imaging," Proc. European Conference on Antennas and Propagation (EuCAP), Prague, 26-30 March 2012.

[6] Helbig M, Hein M, Herrmann R, et al.: "Experimental Active Antenna Measurement Setup for UWB Breast Cancer Detection," Proc. Intern. Conference on Ultra-Wideband (ICUWB), Syracuse, USA, 17-20 Sept. 2012.

[7] Hein M, Helbig M, Kmec M, et al:, "Ultra-Wideband Active Array Imaging for Biomedical Diagnostics" Proc. Intern. Conference on Electromagnetics and Advanced Applications (ICEAA), Cape Town, South Africa, 2-7 Sept. 2012.

[8] Sachs J: "Handbook of Ultra-Wideband Short-Range Sensing: Theory, Sensors, Applications", WileyVCH Verlag, 2012

[9] Kmec M, Helbig M, Sachs J, et al., "Integrated ultrawideband hardware for MIMO sensing using pnsequence approach," Proc. Intern. Conference on Ultra-Wideband (ICUWB), Syracuse, USA, 17-20 Sept. 2012.

[10] Lazebnik M, Madsen EL, Frank GR, et al.: „Tissuemimicking phantom materials for narrowband and ultrawideband microwave applications" Physics in Medicine and Biology, vol. 50, 2005, 4245-4258.

[11] Lazebnik M, Popovic D, et al.: „A large scale of the ultrawideband microwave dielectric properties of normal, benign and malignant breast tissues obtained from cancer surgeries," Physics in Medicine and Biology, vol. 52, 2007, 6093-6115.

\section{Acknowledgment}

This work was supported by the German Science Foundation (DFG) in the framework of the priority program SPP1202 "UKoLoS", project "ultraMEDIS". 